\title{
CHRONIC GENERALIZED MYOCARDIAL ISCHÆMIA WITH ADVANCED CORONARY ARTERY DISEASE
}

\author{
BY \\ W. F. M. FULTON* \\ From the Department of Cardiology, Royal Infirmary, Edinburgh \\ Received September 12, 1955
}

\begin{abstract}
A disparity often exists between the extent of myocardial damage and the severity of the underlying coronary artery disease. Only where pathological examination has been supplemented by detailed injection studies is it possible to provide satisfactory interpretation of the changes found. From such studies the importance of collateral blood supply through enlarged anastomotic channels within the heart has become better appreciated in recent years.

Obliterative disease of the major coronary arteries, together with secondary changes in the smaller vessels, may result in the original coronary tree becoming distorted in a great variety of ways. Nevertheless certain basic trends in anastomotic development may be recognized. When the arterial changes are widespread and of extreme degree a characteristic vascular pattern and pathological picture may appear.

From an investigation of coronary artery disease, using a technique that included stereoarteriography, four closely similar cases have been selected for presentation. These cases illustrate the extensive nature of the vascular changes that may be found in the subendocardial plexus of the left ventricle. No reference to the importance of this plexus in disease has so far been found in previous records; nor does the characteristic vascular pattern now to be described appear to have been mentioned hitherto.

The clinical and pathological findings broadly correspond to the features described in " acute coronary insufficiency" (Friedberg and Horn, 1939). The characteristic vascular pattern found in these cases is considered in relation to the distribution of focal necrotic lesions in the inner layers of the left ventricle, and also in relation to the freedom from massive myocardial infarction, notwithstanding severe obliterative coronary artery disease.
\end{abstract}

\section{MATERIAL AND MethodS}

The four cases to be presented are part of a series of 26 cases of advanced coronary artery disease which have been investigated post-mortem by a special technique. Thirty-six controls were examined of which 18 were patients with cardiac disease other than coronary artery disease; the remainder were cases without cardiac abnormality or with only minor departures from normality.

Each case was subjected to an analysis that included injection of the coronary arteries with a radio-opaque medium, followed by stereo-radiography of the intact heart and of the partially dissected heart. It is intended to publish the details of the method, but for the purpose of this presentation it may be pointed out that certain features of the technique were important in revealing

\footnotetext{
* Research Assistant in Cardiology, University of Edinburgh, now Lecturer in Materia Medica and
} Therapeutics, University of Glasgow. 
the changes described and in making their demonstration readily recordable. These features included (a) a special modification of radiography which eliminated soft-tissue shadow; $(b)$ a method of partial dissection of the heart, incorporating the use of cross-sections of the ventricles, both for radiography and for inspection; and (c) the emphasis placed on stereoscopy of all arteriograms. A few points in technique are discussed in more detail below because of their particular relevance to the demonstration of the changes found.

(1) The injection of bismuth-gelatin was made under controlled pressure. A standard pressure of $150 \mathrm{~mm}$. $\mathrm{Hg}$ was used for the greater part of the series, including the third and fourth cases presented in this communication. The first two cases, however, occurred early in the investigation before the technique was completely standardized. In the first, a pressure of $180 \mathrm{~mm} . \mathrm{Hg}$ was used and in the second $200 \mathrm{~mm}$. Hg for part of the injection. It is felt that this increase in pressure has had no important influence on the findings.

(a) Other cases early in the series were subjected to the same order of pressure and did not show the changes found in these two cases.

(b) The second case in which the vascular changes were least was subjected to the highest pressure.

(c) The changes in the two later cases were essentially similar to the changes found in the first two.

(d) There was no evidence in the arteriograms or in the microscopic preparations of artefact distension of vessels.

(e) Other workers have used pressure ranges of the same order, and higher, without reporting risk of damage to vessel walls and without describing the same features.

(2) The myocardium was sliced at intervals to form transverse sections of both ventricles. In this way the distribution of changes was readily appreciated. The naked-eye appearances were confirmed by multiple histological sections. These included the entire circle of the left ventricle, and most of the right, at a representative level approximately half-way between the atrioventricular groove and the apex. It is on the basis of these sections stained with hæmatoxylin and eosin and by Verhoeff-or Weigert-and van Gieson methods that the diagrams of the distribution of ischæmic changes are drawn.

(3) Measurements of coronary ostia were made by the gentle insertion of curved glass probes of graded size prior to the introduction of the cannulæ.

(4) The heart weights quoted have had $30 \mathrm{~g}$. subtracted from the recorded weights in order to make correction for the added weight of bismuth-gelatin mass.

(5) Red and blue pigments were incorporated in the medium before injection into the right and left coronary arteries respectively.

\section{CASE RePORTS}

Case 1. A. MacD., a woman aged 67 years. Civil servant. No. 315/52. A single woman of middle height and moderate obesity, she had suffered from angina of effort for thirteen years, during which there was progressive increase in the frequency and severity of attacks. Although at times there were temporary episodes of increased severity, the general course was unremittent. Five years before death, cervical sympathectomy was performed with some temporary reduction in the severity of the pain but not of the disability. Death was due to mediastinitis following the removal of a carcinoma of the thyroid. On one occasion only did the duration of an anginal attack last longer than thirty minutes and that was nine years before her death. Despite numerous severe attacks of angina there was no instance in which myocardial infarction could be diagnosed with certainty on clinical or electrocardiographic grounds.

Findings at necropsy. The heart was enlarged as the result of left ventricular hypertrophy and gross increase of epicardial fat. There was no valvular abnormality, and no hypertension had been observed during the last few years of life.

The myocardium of the left ventricle showed widespread patchy fibrosis of the inner zone of the 
entire left ventricle. There was no naked-eye evidence of recent infarction, but scanty areas of focal necrosis were found on histological examination, likewise in inner zonal distribution.

The coronary arteries showed gross atherosclerotic disease with severe narrowing of two, and occlusion of one, main branch. In addition, atherosclerosis of the aorta was accompanied by severe narrowing of both coronary ostia. Large-scale superficial anastomoses formed communications between the epicardial vessels, but the greatest degree of anastomotic development was found in the inner zone of the entire left ventricle. That this zone of vascularity was indeed a dense network of anastomotic channels was demonstrated by stereoradiography; and that filling took place from both coronary vessels was confirmed by the intimate intermingling of pigments in all channels.

Case 2. J. E., a man aged 68 years. Retired excess luggage clerk. For twelve years this man suffered from relentlessly progressive, severe angina, provoked by slight exertion. The onset of the illness was heralded by pain which lasted for one hour and was accompanied by sweating and vomiting. There was a second episode of enduring pain five years later. For three years before death pain had been occurring also at rest.

Increased severity and frequency of anginal attacks in the terminal six months culminated in fatal " status anginosus". Multiple electrocardiograms had been taken over a period of years, and until two days before death there had been no record that suggested myocardial infarction. This last record suggested anteroseptal infarction, which was not, however, confirmed at necropsy.

Although the blood pressure recorded during life was of the order of 200/100, the heart showed no enlargement. The myocardium showed inner zonal fibrosis of the left ventricle and a few scattered areas of focal necrosis. An advanced degree of coronary artery disease was present, with severe narrowing of the right coronary and left circumflex arteries, together with stenosis of both coronary ostia. At no point was there evidence of recent change in the coronary arteries; there was no recent occlusion or intimal hæmorrhage. Increase in the arterial channels was noted in the deeper layers of the left ventricle, with increased prominence of anastomoses in this area as well as elsewhere in the heart.

Case 3. B. M. was a married woman of 73 years, of small build and poor nutrition. There had been progressively increasing effort pain over a period of ten years with angina also at rest for the last two years. Except terminally, attacks of pain were always of short duration and were relieved by rest or trinitrin.

Four weeks before death there was enduring pain which recurred two weeks later with greater severity, and she was admitted to hospital in shock as the result of recent myocardial infarction. Electrocardiograms suggested that this was anteroseptal in distribution. Her condition deteriorated and she died two weeks after admission.

At necropsy widespread atherosclerosis was found. There were cerebrovascular changes; carcinoma of the stomach, without metastasis, was an incidental finding. The heart was enlarged only as the result of fat, and was the seat of advanced coronary disease. In addition both coronary ostia were stenosed. There was highly developed inter-arterial anastomosis, affecting all areas but with greatest intensity in the deep zone of the entire left ventricle. The "inner shell" of the left ventricle showed speckled infarction, composed of small focal areas of necrosis, none larger than $3 \mathrm{~mm}$. in diameter. Fibrosis was also found in the same zone. The outer and subendocardial layers were not involved in the acute changes. No direct precipitating cause for infarction was found; in particular there was no recent occlusion of a coronary vessel. The terminal episodes were of the nature of acute coronary insufficiency.

Case 4. J. S. A housewife, aged 70, had suffered from anginal pain on slight effort for ten years. By keeping within her limits distressing pain was avoided and episodes never lasted longer than ten minutes. At no time was there clinical evidence of infarction except terminally. In latter months the condition was more severe. She was known to have suffered from hypertension for at least two years, with a blood pressure of $200 / 140 \mathrm{~mm}$. $\mathrm{Hg}$. Nineteen hours before her death she developed severe enduring sternal pain requiring morphine for its relief, and she was admitted to hospital in a state of shock. 
At necropsy severe atherosclerosis of the aorta and coronary arteries was found. The heart was enlarged mainly from left ventricular hypertrophy and on section a zone of infarction was seen to encircle the inner half of the entire left ventricle. The appearances were consistent with an infarct of less than twenty-four hours' duration and which was composed of multiple focal areas. The coronary arteries showed no acute changes, in particular no recent occlusion, to account for the development of infarction; but they were the seat of gross atherosclerotic disease and both coronary ostia were stenosed.

\section{SUMMARY OF FINDINGS IN THE Four CASES}

A close similarity was found both in positive and negative findings in these four cases, and some of the features are summarized in Tables I and II.

TABLE I

Clinical features of Four Patients with Prolonged Progressive Myocardial Ischemia

\begin{tabular}{|c|c|c|c|c|c|c|c|c|c|c|}
\hline \multicolumn{7}{|c|}{ Case number } & \multirow{2}{*}{$\frac{1}{\underset{67}{F}}$} & \multirow{2}{*}{$\begin{array}{c}2 \\
65 \\
M \\
\end{array}$} & \multirow{2}{*}{$\begin{array}{c}3 \\
73 \\
0+0\end{array}$} & \multirow{2}{*}{$\begin{array}{c}4 \\
70 \\
F \\
7+1\end{array}$} \\
\hline $\begin{array}{l}\text { Age } \\
\text { Sex } \\
\text { Parity }\end{array}$ & $\begin{array}{l}\cdots \\
\cdots \\
\cdots\end{array}$ & $\begin{array}{l}\cdots \\
\cdots \\
\cdots\end{array}$ & $\begin{array}{l}\cdots \\
\cdots \\
\cdots\end{array}$ & $\begin{array}{l}\cdots \\
\cdots \\
\cdots\end{array}$ & $\begin{array}{l}\cdots \\
\cdots \\
\cdots\end{array}$ & $\begin{array}{l}\cdots \\
\cdots \\
\cdots\end{array}$ & & & & \\
\hline \multicolumn{6}{|c|}{$\begin{array}{l}\text { Duration of history (years) } \\
\text { Usual duration of attack (minutes) } \\
\text { Frequency } \ldots \\
\text { Longest attack apart from terminal episode }\end{array}$} & $\begin{array}{l}\cdots \\
\cdots \\
\cdots\end{array}$ & $\begin{array}{l}13 \frac{1}{2} \\
2-3 \\
20 / \text { day } \\
30 \text { min. }\end{array}$ & $\begin{array}{l}12 \frac{1}{2} \\
2-3 \\
19 / \text { day } \\
60 \mathrm{~min}\end{array}$ & $\begin{array}{c}10 \\
\text { "A few", } \\
\text { " Daily " } \\
\text { "Always } \\
\text { short " }\end{array}$ & $\begin{array}{l}10+ \\
10 \\
\text { "Frequent } \\
10 \text { min. }\end{array}$ \\
\hline
\end{tabular}

TABLE II

Pathological features of the Coronary Arteries in Four Patients with Progressive Myocardial IsChemia

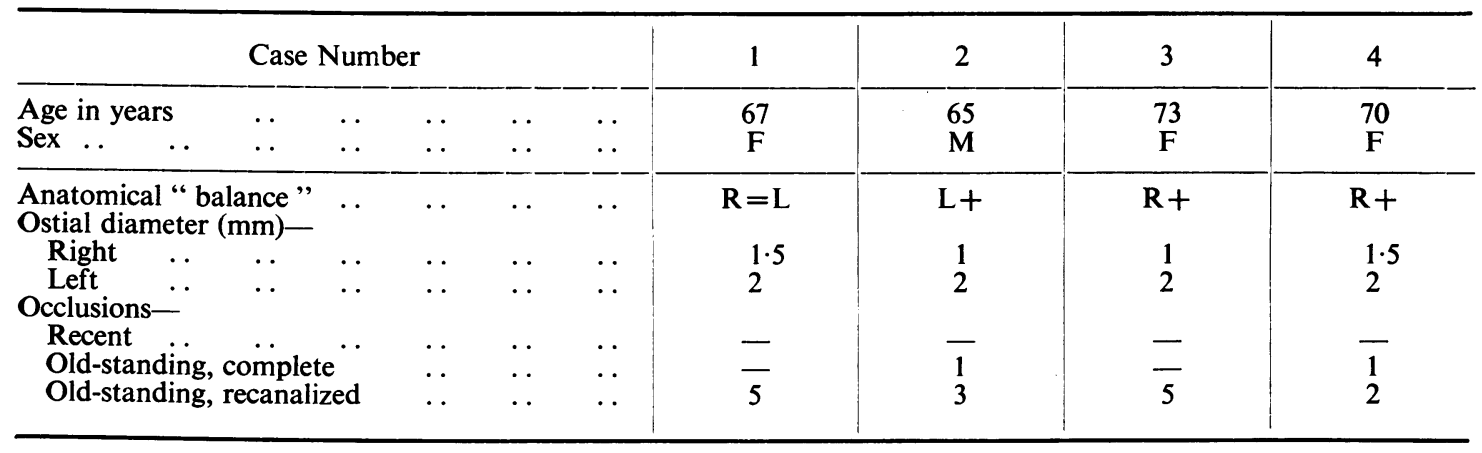

In each case there was a long history of effort pain, and in two pain occurred latterly also at rest. Although angina was severe in three cases, it is felt that the significant feature in this group was not so much the severity of the pain but the relentlessly progressive course, uninterrupted by remissions, and of long duration. Exacerbations occurred but clinically recognized episodes of myocardial infarction were absent except as a terminal event.

In each case there was advanced coronary disease and, in addition, there was also narrowing of both coronary ostia. The blood Wassermann reaction had been negative in life and no evidence was found post mortem of syphilitic involvement of the aorta.

Distributed between the four cases there were two instances of complete occlusion of a main 
arterial stem and fifteen of severe narrowing (i.e. less than $1 \mathrm{~mm}$. in diameter). Detailed investigation suggested that the latter represented recanalization of old occlusions. There was no instance of recent occlusion.

In no case was there evidence of recent or old-standing massive myocardial infarction in the territories of narrowed or occluded arteries. On the other hand, recent myocardial damage could be recognized on gross examination in two cases, and was in the form of multiple focal areas of necrosis measuring $1-3 \mathrm{~mm}$. in diameter, which in aggregate formed a ring of infarction as seen in cross-section throughout the inner shell of the left ventricle. The immediately subendocardial layer was not affected. In the other two cases, isolated areas of focal necrosis in inner zonal distribution were found on histological examination. In no case were these changes the result of recent occlusion.

Fibrosis was of diffuse patchy type and probably represented previous episodes of focal necrosis. It was mainly confined to the inner zone of the left ventricle and likewise spared the immediately sub-endocardial layer. The distribution of focal necrosis and fibrosis in the four cases is shown in Fig. 1. A section through the interventricular septum of Case 4 is shown in Fig. 7 in order to demonstrate the coincidence of vascular and fibrotic changes in the same picture.

\section{CASE 1}

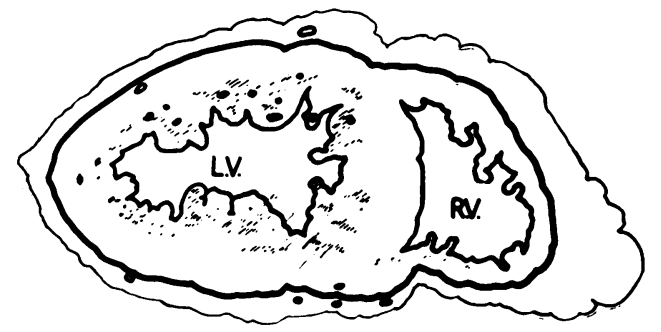

CASE 3

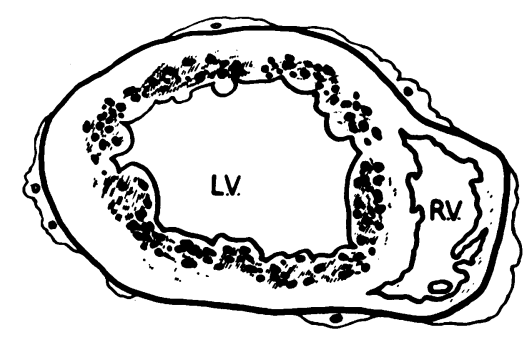

CASE 2

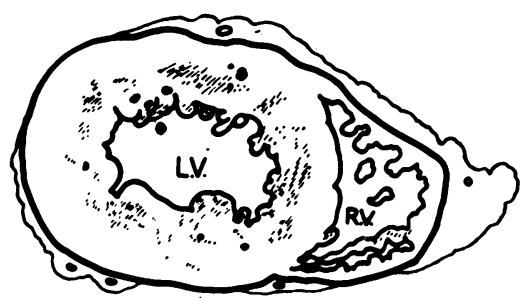

CASE 4

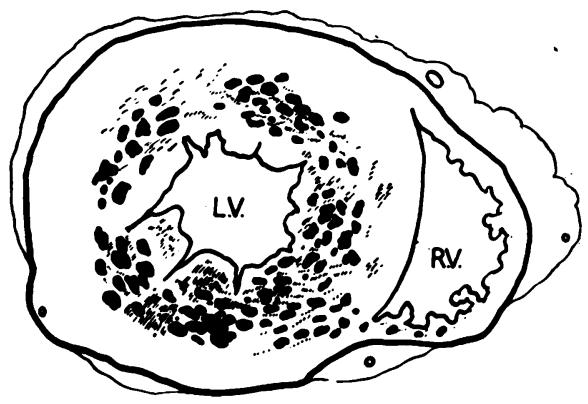

- Focal necrosis

保 "II Frosis

wren

FIG. 1.-Diagram of transverse sections through the ventricles, showing the distribution of recent and old-standing myocardial damage.

No immediate precipitating factor for the development of necrosis was found. Only in the first case was death directly attributable so far as is known to a factor other than coronary disease, although in the third case cerebrovascular disease and the debilitating effects of gastric carcinoma doubtless had some influence. 
Intimal hæmorrhages occurred in two of the cases but without apparently having caused significant narrowing of the lumen. All were intramural in position and of small size, and their presence was unaccompanied by thrombosis. In no case was there valvular disease or congenital abnormality.

\section{Description of the Vascular Pattern in Chronic Generalized MYOCARDIAL ISCHEMIA}

The characteristic vascular change that was found in the four cases described is shown in Fig. 2. In this radiograph of an intact injected heart, the "vascular density" in the central area of the left ventricle forms a striking contrast to the pattern seen in a slightly enlarged but otherwise normal heart (Fig. 3). When the paired arteriograms from the abnormal cases were viewed stereoscopically it was seen that the dense central area was composed of a network of dilated inter-communicating channels which occupied the entire inner zone of the left ventricle.

The position of this network may be demonstrated in two dimensions by taking transverse sections through the ventricles (Fig. 4 and 5). In these the contrast of the distribution of arterial vascularity in the presence of disease to the condition found in health may be appreciated by comparison with the section of a normal heart (Fig. 6). The distribution of the vascular changes will later be correlated with the myocardial findings.

As might be expected, dilated anastomoses were also found on the surface of the heart. These were situated at many areas but were especially large in the neighbourhood of the apex and in the atrio-ventricular groove. These are common sites for anastomoses to be found in the presence of coronary artery disease.

In circumstances where all major branches of the coronary arteries have been affected by atherosclerotic disease of the severity and chronicity found in these cases, and where widespread dilatation of anastomoses has likewise reached an extreme degree, it is evident that all semblance to the original coronary " tree " has been lost. The tree has been replaced by a network.

When this stage has been reached the main coronary arteries have lost most of the local importance formerly held by them in regard to the blood supply of their individual territories, and occlusion of major branches can occur, as in the cases reported, without the development of local myocardial infarction. Indeed, it may be that once the coronary circulation has been converted into such a network, regional (as opposed to zonal) myocardial infarction becomes anatomically impossible. On the other hand, every obstruction to a main vessel presumably introduces further resistance to total flow through the coronary network, and it is possible that some of the exacerbations that occurred in the clinical courses of the patients described were brought about in this way.

In all grades short of that producing myocardial infarction, ischæmia appears to promote the dilatation of the smaller coronary vessels, including the interarterial communications. The flow of blood from a relatively well provided area to the territory of an artery that has become narrowed may be assisted by the vasodilatation that takes place under the influence of anoxia (Gregg, 1950). Moreover, increased blood flow itself has evidently a dynamic influence on enlargement of arteries.

Myocardial ischæmia of a degree insufficient to cause massive structural damage may yet act as a potent stimulus to anastomotic enlargement, provided that it is operative for a sufficiently long period of time. In the cases reported the long histories of anginal pain bear witness to the duration and severity of myocardial ischæmia. The finding that both coronary ostia were narrowed in all cases, and in addition that all major coronary arteries were extensively diseased, suggests that myocardial ischæmia must inevitably have been generalized. All the pathological findings are in keeping with this concept of chronic generalized myocardial ischæmia.

It is submitted that the conversion of the coronary tree into an arterial network provided the anatomical basis upon which structural myocardial damage was largely prevented or delayed, and 


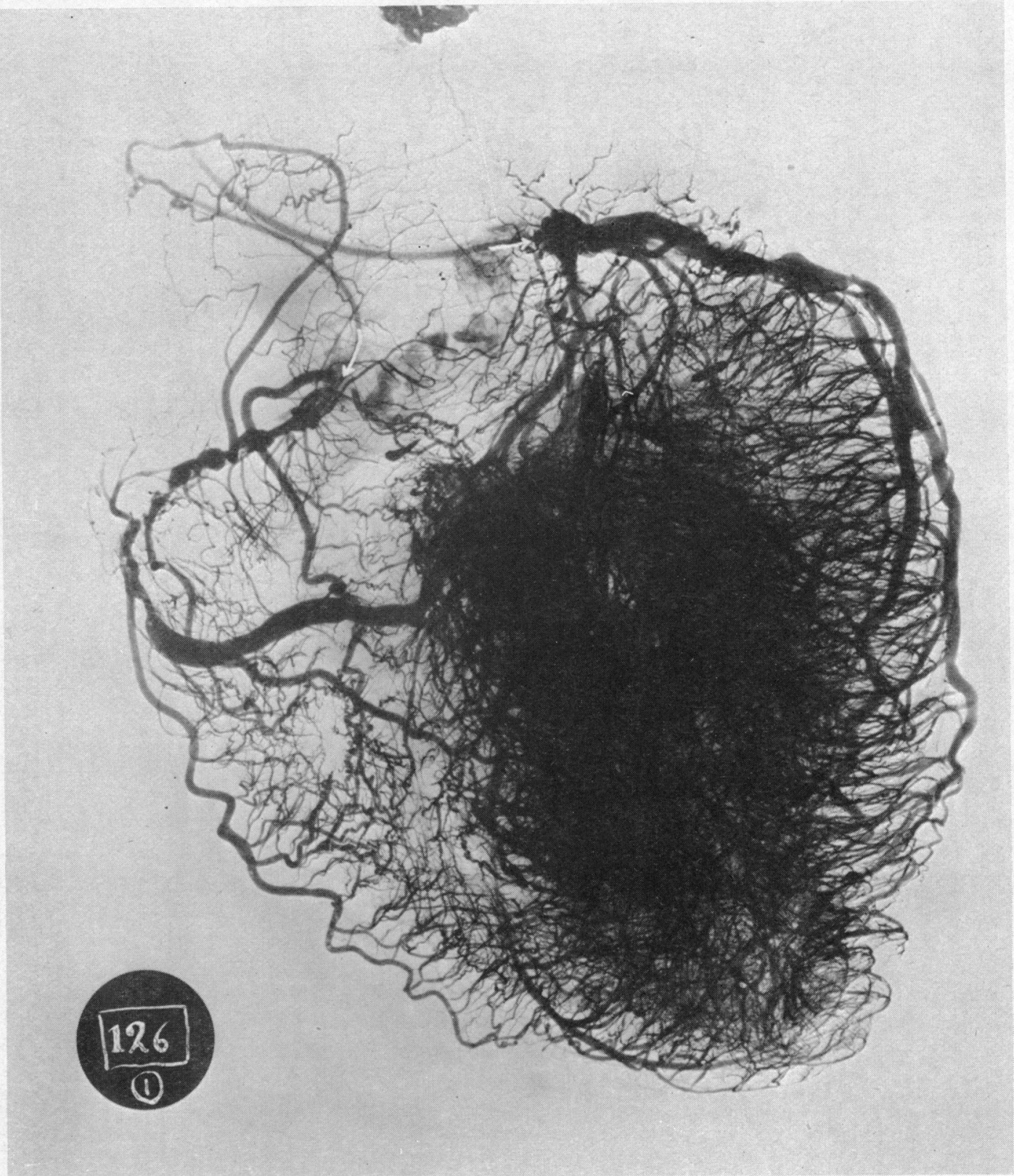

FIG. 2.-Arteriogram of the entire heart, showing the characteristic vascular pattern found in chronic generalized myocardial ischæmia. Case 4. 




Fig. 3.-Arteriogram of the entire heart, showing normal appearances in a control case. 


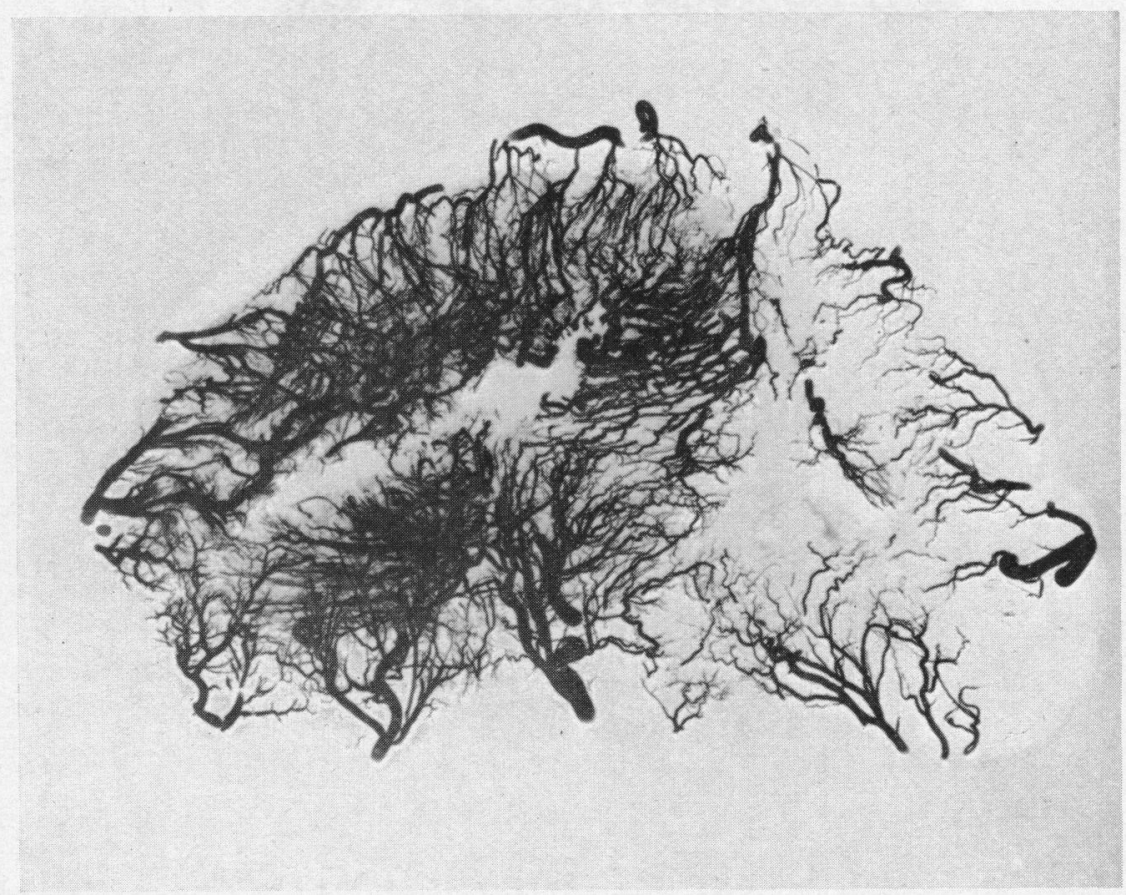

FIG. 4.-Arteriogram of transverse section through the ventricles. Case 1.

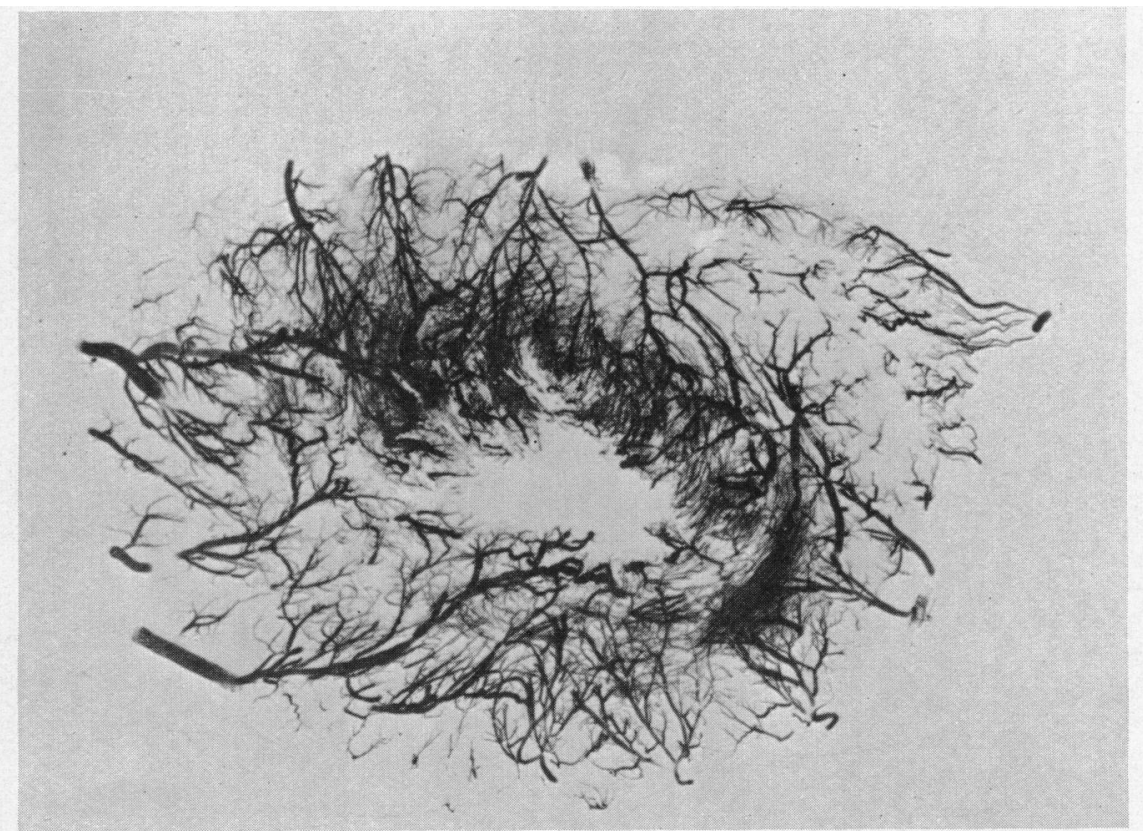

FIG. 5.-Arteriogram of transverse section through the ventricles. Case 4. 


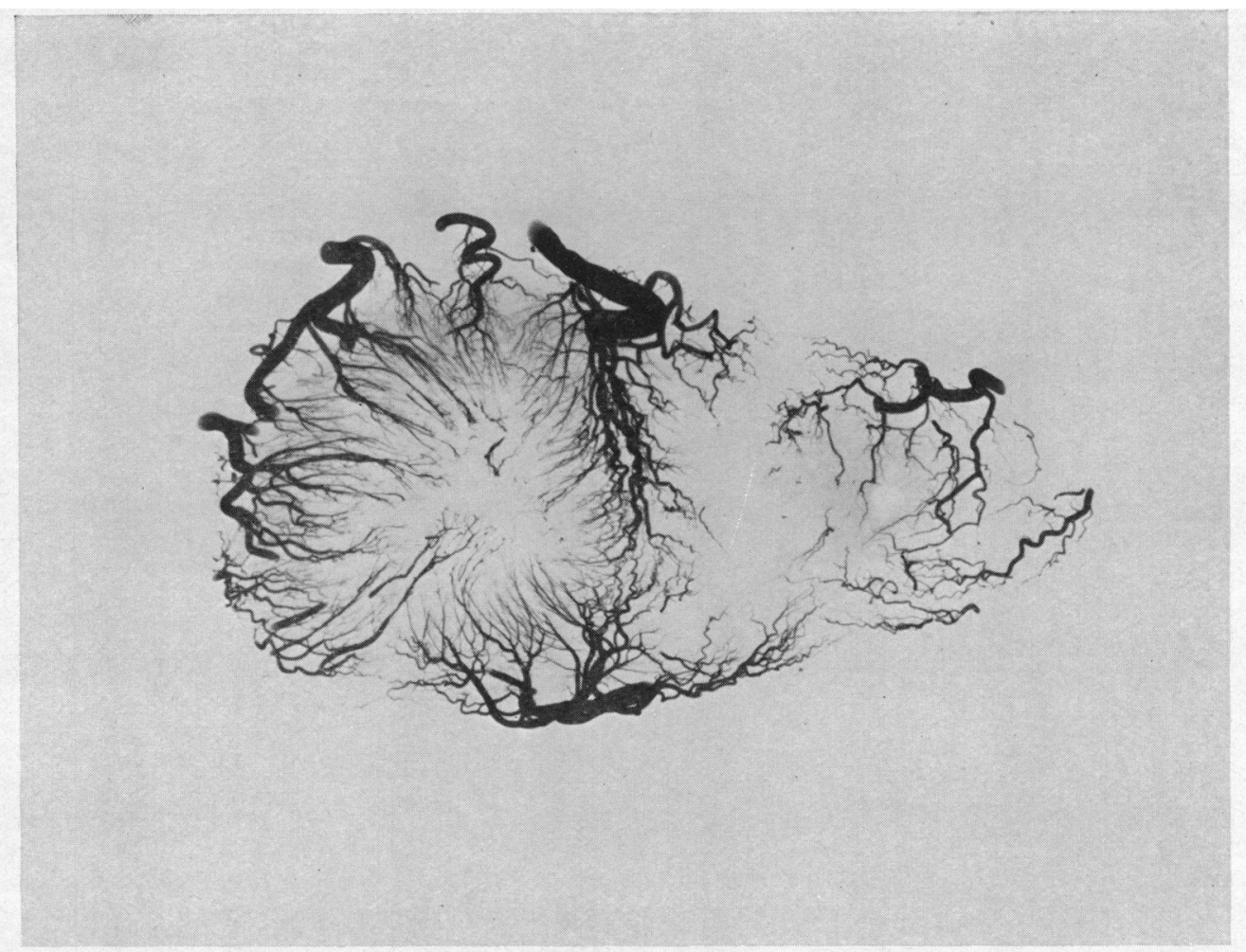

Fig. 6.-Arteriogram of transverse section through the ventricles of a normal heart.

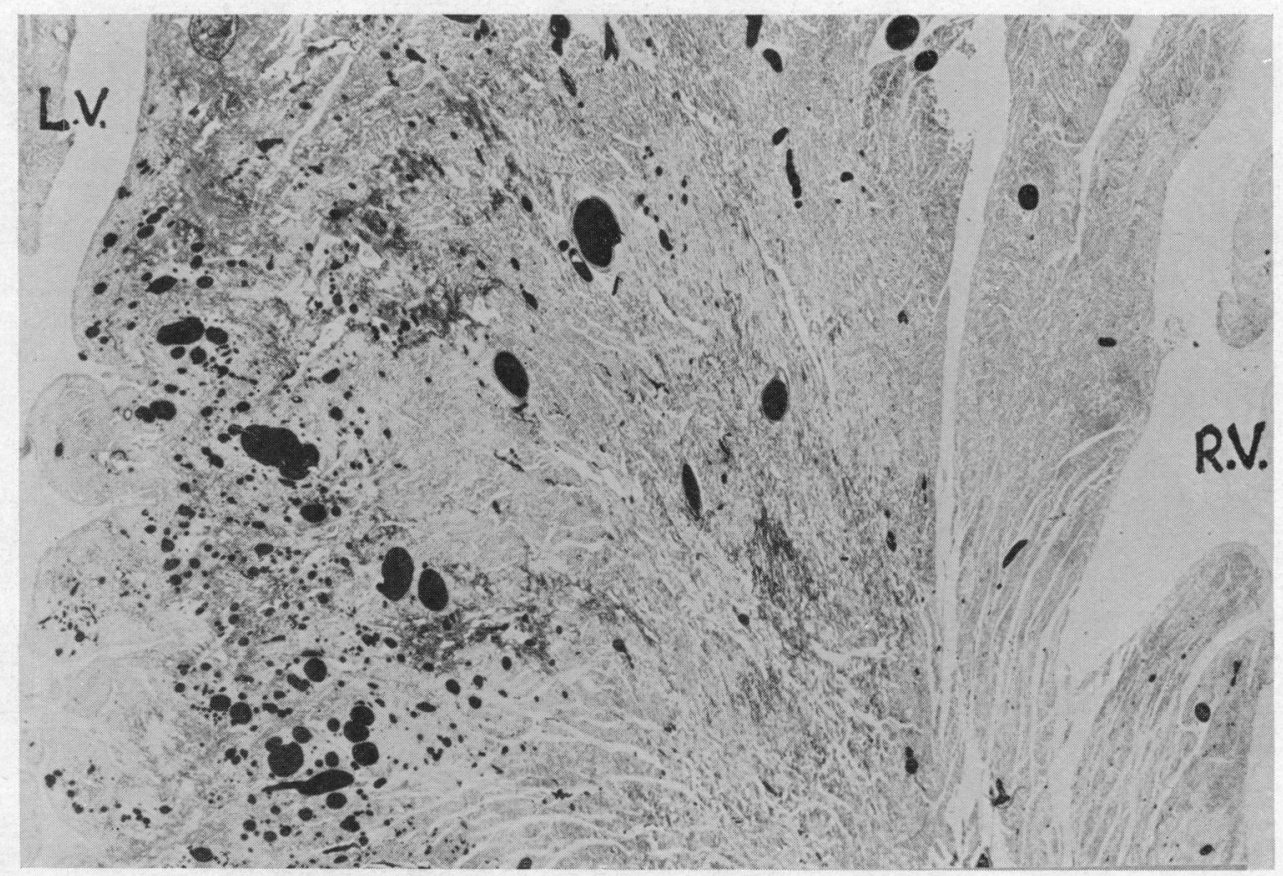

FIG. 7.-Transverse section through the interventricular septum stained by van Gieson's method. Fibrosis is seen mainly on the left ventricular side of the septum. The injection medium within the arteries appears black. Case 4. 
which conditioned the focal character and inner-zonal distribution of myocardial necrosis when it occurred. These last points will now be considered in more detail.

\section{Regional OR ZoNAL MyOcARdial INFARCTION}

The myocardial damage that results from ischæmia may be regarded as falling into two main types according to the manner in which the lesions are distributed. In their typical forms these two types of myocardial infarction can be readily distinguished but intermediate forms exist which are less clearly defined. The distinction between regional and zonal myocardial infarction is a useful one, for, as will be seen, the distribution of the lesions is closely related to the underlying changes in the coronary circulation.

Regional Myocardial Infarction. In many cases of coronary artery disease the obliterative changes fall mainly on one major artery. When myocardial infarction follows the occlusion of this artery, the myocardial damage is restricted to the territory of the affected vessel. The extent of the damage within that region may, however, vary from massive "through and through " infarction of almost the entire anatomical territory to slight damage in the form of a few small areas of necrosis. These small areas of damage tend to be restricted to the deeper layers of the left ventricle. These points are well established and are illustrated in Fig. 8, A and B. A factor that largely determines the extent of myocardial damage resulting from a particular occlusion is the state of the collateral circulation to the affected area that is immediately available at the time of occlusion.

Inner Zonal or "Sub-endocardial "Infarction. The occurrence of multiple focal areas of necrosis in ischæmic heart disease is likewise well recognized and its association with the clinical conditions of " acute coronary insufficiency" has been pointed out by Büchner (1939) and by Friedberg and Horn (1939), Master et al. (1941) and Horn et al. (1950). These observers recognize that the focal ischæmic lesions are distributed predominantly to the deeper layers of the muscle of the left ventricle. The present series is in entire agreement with their findings. On the other hand, in the published cases of acute coronary insufficiency which have been quoted, injection techniques were not employed in the examination of the coronary arteries. Accordingly, no correlation with the vascular pattern could be made. Further, no published description of the vascular pattern found in the present series has been discovered. As already indicated, this vascular pattern is felt to be the key to the other pathological changes.

\section{The Relationship Between Anastomoses and the Relief of Ischæmia}

It has been stated by Zoll et al. (1951) that the development of increased arterial anastomosis in the coronary circulation is a compensatory change, which indeed it is. It may be argued, however, that the beneficial effects of anastomotic development are fundamentally fortuitous; and that the favourable outcome that may result from anastomotic enlargement bears no causal relationship to the processes conditioning that enlargement. This point is made in connection with the four cases under presentation, because they provide clear demonstration that vascular " adaptations" in the form of dilated anastomoses occur as a secondary effect of ischæmia, and that these changes take place quite independently of the capacity of the " friendly neighbouring arteries ", as Herrick called them, to provide relief (Herrick, 1912). Indeed, in the present investigation the greatest complexity of anastomotic development has been found in those hearts in which enlargement of communicating vessels has failed to relieve ischæmia; where, so to speak, each artery has been so compromised by disease that it can barely carry sufficient blood for the basic needs of its own territory, and is therefore not in a condition to afford charity for its neighbours. In these circumstances, where inflow of blood to the whole organ has been reduced at source, internal rearrangement of channels of supply can at the best achieve only a more uniform distribution of poverty. This appears to have been the situation in the four cases described. It is understandable 

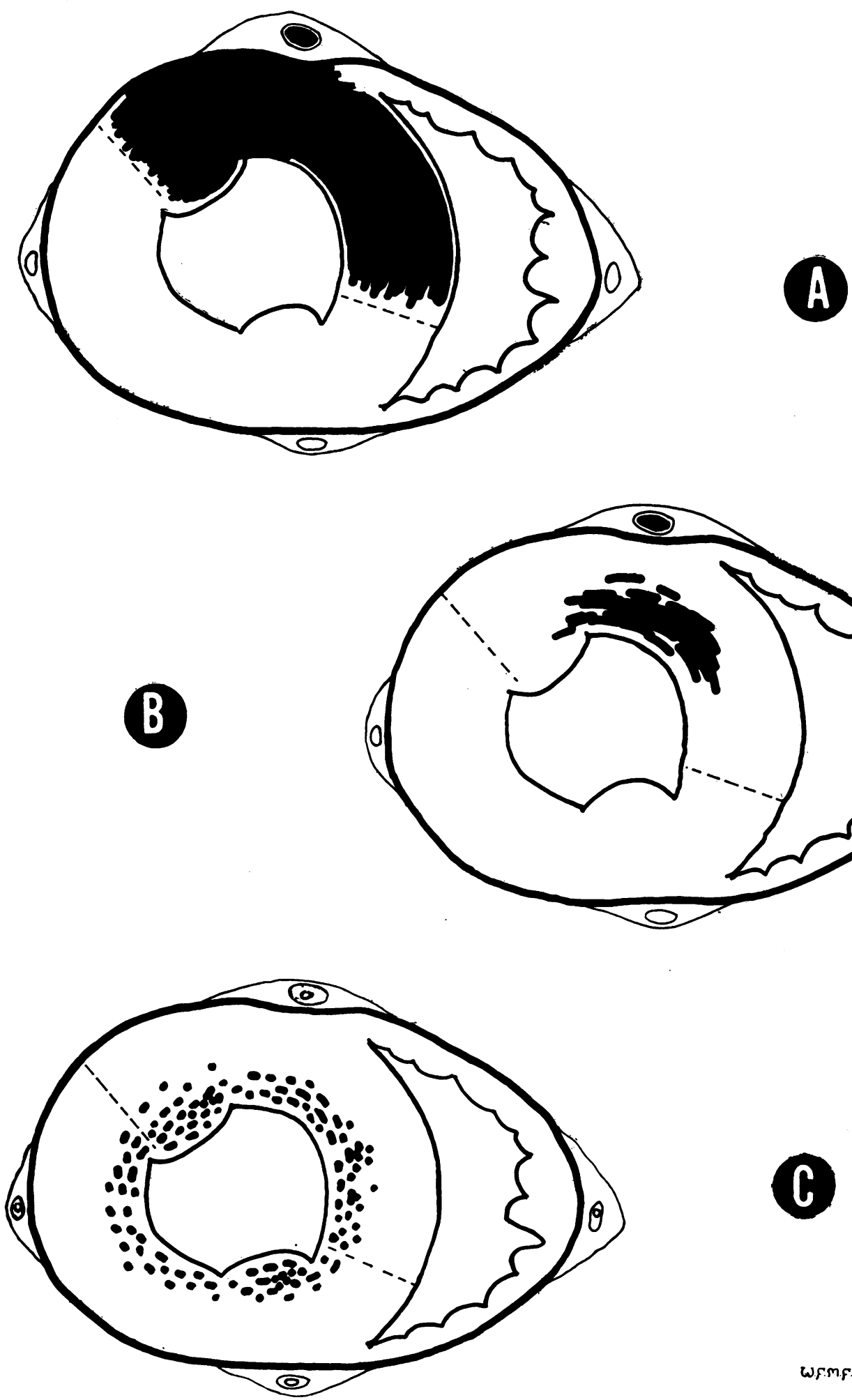

Fig. 8.-Diagram illustrating the distribution of damage in regional and zonal infarction. (A) Regional infarction, massive, "through and through". (B) Regional infarction, sub-total. (C) Inner zonal, "ring "infarction (focal necrosis). 
that in such circumstances the least favourably situated areas should have suffered first, and that these should have lain farthest from the source of blood supply; and moreover should have lain in those layers of the left ventricle to which delivery of blood during systole is impeded to the greatest extent by extravascular compression.

\section{Factors Influencing the InNer Zonal Distribution of Focal Necrosis}

The most obvious factor conditioning the inner zonal distribution of focal necrosis in acute coronary insufficiency is one of distance from supply. This was the explanation which first appealed to Friedberg and Horn (1939). The demonstration of the conversion of the coronary " tree " into a network of the order that has been revealed in this investigation puts this explanation on a sound anatomical basis.

Other explanations have been put forward and mention is made of two of them. From the observation of Johnson and Di Palma (1939) that a gradient of pressure exists during systole which diminishes from the inner to the outer layers of the left ventricle, it was deduced (Horn et al., 1950) that the inner layers are therefore more susceptible to oxygen deprivation. These authors also offer in explanation the evidence derived from acute experiments on the dog in which it was observed that the sub-endocardial layer of the left ventricle seemed to have a poorer collateral circulation than the epicardial portions (Prinzmetal et al., 1948). It is felt that such evidence cannot be directly applicable to the human heart in the presence of advanced coronary artery disease. In this connection it may be observed that of the manifestations of coronary artery disease, " acute " coronary insufficiency may well have the most chronic pathological basis.

It is quite possible that the vascular pattern described in this presentation is present in at least the majority of cases of acute coronary insufficiency, when this condition is primarily the result of advanced coronary artery disease, and that this vascular pattern in turn conditions the nature of the clinico-pathological syndrome. The small size of this series, the absence of previous descriptions of the vascular pattern, and the lack of evidence in regard to the smaller vessels in the reported cases of acute coronary insufficiency, prevent certainty on this point.

\section{SummaRY AND CONCLUSIONS}

A series of 26 cases of advanced coronary artery disease with 36 controls were investigated after death by a technique that included stereo-arteriography.

Four cases were selected for special description: they had in common a long history of relentlessly progressive anginal pain, widespread severe coronary artery disease, narrowing of both coronary ostia, inner-zonal distribution of ischæmic changes in the left ventricle, and a characteristic pattern of adaptation of the smaller vessels of the coronary circulation.

Anastomotic development was of an advanced degree and affected the sub-endocardial arterial plexus of the left ventricle with special emphasis.

Terminal myocardial damage was in the form of focal necrosis, which was extensive in two cases, and which was in no case precipitated by recent coronary artery occlusion. Fibrosis in the same distribution was thought to be the sequel of earlier episodes of focal necrosis. The pathological findings were consistent with the features described in acute coronary insufficiency. Despite evidence that thrombotic occlusion had been of frequent occurrence, there was no definite regional infarction. It is postulated that the conversion of the coronary circulation into a network of dilated channels had removed the anatomical basis upon which regional infarction could have occurred.

Factors determining the distribution of focal necrosis to the inner layers of the entire left ventricle are discussed and it is suggested that remoteness from arterial supply is the most likely single factor governing this distribution.

Attention is drawn to the very chronic nature of the background of arterial disease which was found in these cases in which coronary insufficiency was primarily due to coronary atherosclerosis. $2 \mathrm{~A}$ 
Little reference has been found to the importance in disease of the sub-endocardial plexus; and it is believed that the vascular pattern observed in the four cases presented has not previously been described.

The cases reported had all been under clinical observation in the wards of the Cardiological Department of the Royal Infirmary, Edinburgh, under Dr. A. Rae Gilchrist to whom I am indebted for his encouragement and advice. The pathological investigation was undertaken in the Pathology Department of the Royal Infirmary and University of Edinburgh; and to Professor A. Murray Drennan and Dr. R. F. Ogilvie I am deeply grateful for the facilities provided and for their stimulating and critical guidance. The photographic reproductions were carried out in the Department of Photomicrography under Mr. T. Dodds, and facilities for radiography were afforded by Professor J. C. Brash in the Department of Anatomy.

The work was carried out during the tenure of a Research Assistantship in Cardiology in the University of Edinburgh and was sponsored by the Atherosclerosis Committee of the Department of Health for Scotland.

\section{REFERENCES}

Barnes, A. R., and Ball, R. G. (1932). Amer. J. med. Sci., 183, 215.

Büchner, F. (1939). Die Koronarinsuffizienz. Dresden u. Leipzig, T. Steinkopff.

Friedberg, C. K., and Horn, H. (1939). J. Amer. med. Ass., 112, 1675.

Gregg, D. (1950). Coronary Circulation in Health and Disease. Lea and Febiger, Philadelphia.

Gross, L. (1921). The Blood Supply to the Heart. Oxford University Press.

Herrick, J. B. (1912). J. Amer. med. Ass., 59, 2015.

Horn, H., Field, L. E., Dack, S., and Master, A. M. (1950). Amer. Heart J., 40, 63.

Johnson, J. R., and Di Palma, J. R. (1939). Amer. J. Physiol., 125, 234.

Master, A. M., Gubner, R., Dack, S., and Jaffe, H. L. (1941). Arch. intern. Med., 67, 646.

, Horn, H., Grisham, A., and Field, L. E. (1949). Amer. J. Med., 7, 464.

- Dack, S., Horn, H., Freedman, B. L., and Field, L. E. (1950). Circulation, 1, 1302.

Prinzmetal, M., Bergman, H. C., Krueger, H. E., Schwarz, L. L., Simpkin, B., and Sobin, S. S. (1948). Amer. Heart J., 35, 689.

Spalteholz, W. (1907). Verhandl. d. anatomischen Gesell, 24-27, April, 141.

(1924). Die Arterien der Herzwand. Leipzig, S. Hirzel.

Zoll, P. M., Wessler, S., and Blumgart, H. L. (1951). Amer. J. Med., 11, 331. 